HD-THEP-03-24

May 21, 2003

\title{
Supersymmetric Models For Gauge Inflation
}

\author{
R. Hofmann ${ }^{\diamond}$ F. Paccetti Correia ${ }^{\diamond}$ M. G. Schmidt ${ }^{\diamond}$, \\ Z. Tavartkiladze ${ }^{\diamond, 1}$ \\ Institut für Theoretische Physik, Universität Heidelberg \\ Philosophenweg 16, 69120 Heidelberg, Germany
}

\begin{abstract}
We present possible realizations of gauge inflation arising from a $5 \mathrm{D} \mathcal{N}=1$ supersymmetric $U(1)$ model, where the extra dimension is compactified on a circle. A one-loop inflaton effective $4 \mathrm{D}$ potential is generated, with the inflaton being a 'Wilson-line field'. It relies on a SUSY breaking. We first consider SUSY breaking to occur spontaneously within a 'no-scale' model by a non zero $F$-term of the radion superfield which transmits SUSY breaking into the 'visible' sector. As an alternative, we study $D$-term SUSY breaking originating directly from the 5D gauge supermultiplet. Together with the usual KK resummation method, we present a calculation with the world-line formalism. The latter allows one to get the resulting effective potential directly as a sum over all winding modes. For both presented scenarios, the generated effective potentials have suitable forms for realizing successful inflation, i.e. are flat enough and give the needed number of e-foldings. In addition, there is a natural way to get strongly suppressed values for the potentials, which then could be associated with dark energy/quintessence.
\end{abstract}

\footnotetext{
^E-mail addresses: R.Hofmann@, F.Paccetti@, M.G.Schmidt@, Z.Tavartkiladze@ThPhys.Uni-Heidelberg.DE

${ }^{1}$ On leave of absence from Institute of Physics, GAS, Tbilisi 380077, Georgia.
} 


\section{Introduction}

Inflation is an efficient way to solve the cosmological flatness, horizon, and monopole problem [1]. It explains naturally why the visible part of the universe appears to be isotropic on large scales. Field theoretic real-time models of inflation can explain the seeding of structure formation. The experimental data on the spectral properties of primordial density perturbations do not lead to a unique inflation model in field theory. If in a given field theory model the scale of inflation is close to the $4 \mathrm{D}$ Planck scale $M_{P}$ the slow-roll requirements linked to the model's treelevel potential generically are violated by the appearance of gravitationally induced operators. Even for low-scale inflation the coupling of the inflaton to other fields induces radiative corrections to the inflaton potential which are hard to control. This problem is much milder if a symmetry protects the potential. For example spontaneously broken, rigid supersymmetry (SUSY) yields flat potentials [2] for inflaton fields if the scale of SUSY breaking is much lower than $M_{P}$. Another possibility for the generation of radiatively stable, flat potentials is the spontaneous breakdown of a global symmetry at scale $f$. Very recently, this possibility was used to construct Little Higgs theories [3]. Furthermore, spontaneously broken global symmetries may underly the curvaton scenario for the non adiabatic generation of curvature perturbations after inflation [4]. Coming back to inflation itself, it is well known that a small amount of explicit symmetry breaking on top of the spontaneous breakdown opens up the possibility that (quasi) de-Sitter-cosmology can be driven by the associated pseudo Nambu-Goldstone (PNG) fields [5]. Slow roll of these fields is ensured if $M_{P} / f \ll 1$. However, a symmetry breaking above $M_{P}$ is probably beyond a field theoretical treatment. This significantly reduces the appeal of the $4 \mathrm{D}$ PNG model. This problem was recently pointed out in [6], and a resolution in terms of gauge symmetry combined with the assumption of extra, compactified dimensions was proposed. In this context the possible generation of a varying spectral index of the primordial, adiabatic scalar perturbations was investigated in ref. [7]. WMAP data [8] indicate a tilted spectrum.

A 4D effective potential for the Wilson-line phase

$$
\Theta \equiv \int_{0}^{2 \pi R} d x^{5} A_{5},
$$

( $R$ denotes the compactification radius) arises by means of the Hosotani mechanism [9] if charged states are present in a theory. The potential is protected from local quantum gravity corrections due to 5D gauge invariance. Moreover, the scale $f_{\Theta}$, which enters the slow-roll condition $M_{P} / f_{\Theta} \ll 1$, is naturally larger than $M_{P}$ if the effective $4 \mathrm{D}$ gauge theory is weakly coupled. A variant of this model uses a mass $M \gg R^{-1}$ for the charged bulk fluctuations which exponentially suppresses the value of the potential [6] making it a suitable candidate [10] for dark energy i.e. quintessence [11]. To derive the effective 4D potential for $\Theta$ one usually appeals to 'Kaluza-Klein' (KK) regularization, i.e. a Poisson resummation of the KK spectrum 
[12]. This recipe avoids the use of a cutoff which would destroy some of the symmetries of the underlying higher dimensional theory. Recently, KK regularization was intensively disputed, and it was pointed out that SUSY and gauge symmetry in field theory help to make KK regularization consistent but that ultimately it is string theory that provides a physical KK completion in the ultraviolet [13]. It may be helpful in this context to point out that in a world-line formulation of quantum field theory [14] in 5D spacetime no Poisson resummation of the KK tower is needed, since directly a sum over winding modes is introduced in the action [23]

$$
\begin{gathered}
-\int d^{4} x d x_{5} \int_{0}^{\infty} \frac{d T}{T} \sum_{k=-\infty}^{\infty} \int \mathcal{D}^{5} y \exp \left[-\int_{0}^{T} d \tau\left(\frac{\left(\dot{x}_{M}\right)^{2}}{4}+i q \dot{x}_{5} A_{5}+M^{2}\right)\right] \\
\text { with } x_{M}(\tau)=2 \pi R k \frac{\tau}{T} \delta_{M 5}+y_{M}(\tau)+x_{M}, \quad \int_{0}^{T} d \tau y_{M}(\tau)=0
\end{gathered}
$$

(see Appendix B for details). In the non-SUSY case this approach exhibits a 5D UV divergence from the zero-winding sector in the one-loop effective potential which is cut off by restricting the proper-time integration to $T \geq \Lambda^{-2}$.

In a SUSY setting, inflation can only occur if SUSY is broken somehow. The purpose of the present paper is to investigate how in the simple situations of $5 \mathrm{D}$ $\mathcal{N}=1$ SUSY $U(1)$ gauge dynamics SUSY breaking translate into a potential for $\Theta$. We assume the extra dimensional coordinate $y$ to describe a circle. Our results can be used as guidelines for the more realistic cases of non Abelian gauge symmetry and/or a larger number of (orbifolded) extra dimensions. In the next section we introduce our model. We use the formulation of [15] with a chiral radion field $\mathcal{T}$ and consider spontaneous SUSY breaking by a no-scale sector such that $F_{T} \neq 0$. We also consider $D$-term SUSY breaking by assuming that the neutral scalar component of the chiral superfield $\Phi$ in the 5D gauge supermultiplet obtains a profile along the extra dimension. The effect on the spectrum of the KK modes is investigated for both cases. In Sections 3 and 4 we compute the effective one-loop potentials for $\Theta$. In Sec. 5 we present cosmological applications of the models. Sec. 6 has a discussion and an outlook. Appendix A is devoted to an analysis of the KK spectra. It also contains technical details concerning the computation of the effective potential. In Appendix B a calculation of the effective potential is presented which uses the world-line method.

\section{The Model}

\subsection{Set-up of 5D dynamics}

Consider 5D $\mathcal{N}=1$ SUSY $U(1)$ gauge theory formulation [16] with the fifth spatial and flat dimension $y$ compactified on a circle, $0 \leq y \leq 2 \pi R$. We stick to the usual convention that $4 \mathrm{D}$ coordinates are labeled by Greek indices $(\mu=0, \cdots, 3)$. 
$5 \mathrm{D} \mathcal{N}=1$ supersymmetry is equivalent to $4 \mathrm{D} \mathcal{N}=2$ SUSY. In terms of $\mathcal{N}=1$ supermultiplets, the $5 \mathrm{D}$ gauge supermultiplet is $\mathbf{V}_{\mathcal{N}=2}=(\mathbf{V}, \Sigma)$, where

$$
\mathbf{V}=-\theta \sigma^{\mu} \bar{\theta} A_{\mu}+\mathrm{i} \bar{\theta}^{2} \theta \lambda_{1}-\mathrm{i} \theta^{2} \bar{\theta} \bar{\lambda}_{1}+\frac{1}{2} \bar{\theta}^{2} \theta^{2} D
$$

is a $4 \mathrm{D}$ vector superfield, while $\Sigma$ is a chiral superfield

$$
\Sigma=\frac{1}{\sqrt{2}}\left(\Phi+\mathrm{i} A_{5}\right)+\sqrt{2} \theta \lambda_{2}+\theta^{2} F_{\Sigma} .
$$

The 5D Lagrangian $\mathcal{L}_{\mathbf{V}}$ of the pure gauge sector reads [15], [16]

$\mathcal{L}_{\mathbf{V}}=\frac{1}{g^{2}} \int d^{4} \theta\left[\left(\sqrt{2} \partial_{5} \mathbf{V}-\Sigma^{+}\right)\left(\sqrt{2} \partial_{5} \mathbf{V}-\Sigma\right)-\left(\partial_{5} \mathbf{V}\right)^{2}\right]+\frac{1}{4 g^{2}}\left(\int d^{2} \theta W^{\alpha} W_{\alpha}+\right.$ h.c. $)$,

where

$$
\begin{gathered}
W_{\alpha}=-\mathrm{i} \lambda_{1 \alpha}+\left[\delta_{\alpha}^{\beta} D-\mathrm{i}\left(\sigma^{\mu \nu}\right)_{\alpha}^{\beta} A_{\mu \nu}\right] \theta_{\beta}+\theta^{2}\left(\sigma^{\mu} \partial_{\mu} \bar{\lambda}\right)_{\alpha} \\
\sigma^{\mu \nu}=\frac{1}{4}\left(\sigma^{\mu} \bar{\sigma}^{\nu}-\sigma^{\nu} \bar{\sigma}^{\mu}\right), \quad A_{\mu \nu}=\partial_{\mu} A_{\nu}-\partial_{\nu} A_{\mu},\left.\quad W^{\alpha} W_{\alpha}\right|_{\theta \theta}=D^{2}+\cdots .
\end{gathered}
$$

In addition, there are two chiral superfields

$$
\phi=\varphi+\sqrt{2} \theta \lambda_{\phi}+\theta^{2} F_{\phi}, \quad \bar{\phi}=\bar{\varphi}+\sqrt{2} \theta \lambda_{\bar{\phi}}+\theta^{2} F_{\bar{\phi}}
$$

with respective charges $q$ and $-q$. These superfields constitute the 5D hypermultiplet $\phi_{\mathcal{N}=2}=(\phi, \bar{\phi})$. The 5D Lagrangian $\mathcal{L}_{\phi}$ for the matter reads

$$
\mathcal{L}_{\phi}=\int d^{4} \theta\left(\phi^{+} e^{-2 q \mathbf{v}} \phi+\bar{\phi} e^{2 q \mathbf{v}} \bar{\phi}^{+}\right)+\left(\int d^{2} \theta \bar{\phi}\left(M+\partial_{5}-\sqrt{2} q \Sigma\right) \phi+\text { h.c. }\right)
$$

where $M$ denotes a real SUSY bulk mass. Each of the Lagrangians (5), (8) is invariant under $5 \mathrm{D} U(1)$ gauge transformations

$$
\begin{gathered}
\mathbf{V} \rightarrow \mathbf{V}+\frac{1}{2}\left(\Lambda+\Lambda^{+}\right), \quad \Sigma \rightarrow \Sigma+\frac{1}{\sqrt{2}} \partial_{5} \Lambda, \\
\phi \rightarrow e^{q \Lambda} \phi, \quad \bar{\phi} \rightarrow e^{-q \Lambda} \bar{\phi} .
\end{gathered}
$$

Also there is invariance under two $4 \mathrm{D} \mathcal{N}=1$ SUSY transformations. These two supersymmetries are related by an $S U(2)_{R}$ symmetry. The bosonic part of $\mathcal{L}_{\mathbf{V}}+\mathcal{L}_{\phi}$, involving $4 \mathrm{D}$ scalar and auxiliary components, reads

$$
\begin{aligned}
& \left(\mathcal{L}_{\mathbf{V}}+\mathcal{L}_{\phi}\right)^{B}=\frac{1}{2 g^{2}} D^{2}-\frac{1}{g^{2}} \Phi \partial_{5} D+\frac{1}{g^{2}}\left|F_{\Sigma}\right|^{2}+\left|F_{\phi}\right|^{2}+\left|F_{\bar{\phi}}\right|^{2}-q D\left(|\varphi|^{2}-|\bar{\varphi}|^{2}\right)+ \\
& {\left[F_{\bar{\phi}}\left(M+\partial_{5}-q\left(\Phi+i A_{5}\right)\right) \varphi+\bar{\varphi}\left(M+\partial_{5}-q\left(\Phi+i A_{5}\right)\right) F_{\phi}-\sqrt{2} q \bar{\varphi} F_{\Sigma} \varphi+\text { h.c. }\right] .}
\end{aligned}
$$


Eliminating $F$ and $D$-terms from (10), the part $\mathcal{V}$ in (10), which does not contain $4 \mathrm{D}$ derivatives, reads

$$
\begin{gathered}
\mathcal{V}=\frac{1}{2 g^{2}}\left(\partial_{5} \Phi\right)^{2}+\frac{g^{2}}{2} q^{2}\left(|\varphi|^{2}-|\bar{\varphi}|^{2}\right)^{2}+2 g^{2} q^{2}|\varphi|^{2}|\bar{\varphi}|^{2}+ \\
(M-q \Phi)^{2}\left(|\varphi|^{2}+|\bar{\varphi}|^{2}\right)+\left|\left(\partial_{5}-\mathrm{i} q A_{5}\right) \varphi\right|^{2}+\left|\left(\partial_{5}+\mathrm{i} q A_{5}\right) \bar{\varphi}\right|^{2} .
\end{gathered}
$$

If we assume the $\mathrm{VEV} A_{5}$ to be constant in $y$, the Wilson-line phase $\Theta$ generated by a line integration along the entire extra dimension reads

$$
\Theta=2 \pi R A_{5} .
$$

In order to generate a 4D effective one-loop potential for the field $\Theta$ by the Hosotani mechanism [9] SUSY must be broken in some way. We distinguish two cases of SUSY breaking in the following: (i) spontaneous breaking by the dynamics of a no-scale sector, (ii) $D$-term breaking by a non vanishing (initial) value of the VEV of $\Sigma$ 's scalar component $\Phi$ in (4).

We will discuss the physics being potentially responsible for situation (ii) below. As for case (i) some technical remarks are in order. A superspace formulation of the theory $\mathcal{L}_{\mathbf{V}}+\mathcal{L}_{\phi}$ involving a chiral radion superfield

$$
\mathcal{T}=\mathcal{R}+i B_{5}+\theta \Psi_{R}^{5}+\theta^{2} \mathcal{F}_{\mathcal{T}}
$$

has been proposed in ref. [15]. The field $\mathcal{T}$ appears in terms of a factor $\left(\mathcal{T}^{\dagger}+\right.$ $\mathcal{T}) /(2 R)$ for the first term in (8), and in terms of a factor $2 R\left(\mathcal{T}+\mathcal{T}^{\dagger}\right)^{-1}$ and a factor $\mathcal{T} / R$ for the first and second term, respectively, in (5). Upon $\theta$ and $\bar{\theta}$ integration, the elimination of the auxiliary fields by their equations of motion this formulation yields the usual component-field 5D Lagrangian $\mathcal{L}_{V}+\mathcal{L}_{\phi}$. The radion field $\mathcal{T}$ is of the no-scale type [15] and has a flat potential in the $\langle\mathcal{T}\rangle$ direction in string-derived [18] supergravity models [17]. The superfunction $G=-3 \ln K+$ $\ln |g|^{2}, K$ denoting the Kähler potential and $g$ the superpotential, in such 'noscale' [17] ('non minimal' [19] or 'minimal $S U(n, 1)$ ' [20]) models can be written in the form $G=-3 \ln \left(\mathcal{T}+\mathcal{T}^{\dagger}-\left|\phi_{i}\right|^{2}\right)+\ln \left|g_{3}\left(\phi_{i}, \mathcal{T}\right)\right|^{2}$ where $\phi_{i}$ denote chiral fields, $g_{3}$ is a superpotential, and all fields are in Planck units. $G$ is invariant under particular Kähler transformations being related to dilatations. On tree-level noscale models have $\langle\mathcal{R}\rangle=R$ as a sliding scale in a flat potential, and the scale of SUSY breaking $\left\langle\mathcal{F}_{\mathcal{T}}\right\rangle \equiv F_{T}$ is also not fixed. We assume $R$ to be stabilized by some additional dynamics, see for example [21], and appeal to the radiative stabilization of $F_{T}$ as in [25]. With ref. [15] we will discuss in the next section how $F_{T} \neq 0$ affects the spectrum of the charged KK modes whose fluctuations generate a $4 \mathrm{D}$ one-loop effective potential for $\Theta^{1}$.

\footnotetext{
${ }^{1}$ The way the SUSY breaking $F_{T}$ is derived in [15] using a 'compensator field', which drops out of $G$, should be compared in detail with the methods in [20]. A radiatively stabilized VEV $F_{T} \neq 0$ as it arises in gauged SUGRA [25] was mimicked by a flat-space no-scale sector in [15]. In this model a scale $W$ enters the superpotential for a conformal compensator chiral superfield and determines the VEV $F_{T}$. Stabilization is therefore already assumed in this effective description.
} 


\subsection{KK decomposition and SUSY breaking}

Since we compactify on a circle the usual consideration of reflection parity for orbifold compactifications does not apply. The charged scalar fields $\phi$ and $\bar{\phi}$ have the following $\mathrm{KK}$ decompositions

$$
\varphi(x, y)=\frac{1}{\sqrt{2 \pi R}} \sum_{n=-\infty}^{+\infty} \varphi^{(n)}(x) e^{\mathrm{i} \frac{n y}{R}}, \quad \bar{\varphi}(x, y)=\frac{1}{\sqrt{2 \pi R}} \sum_{n=-\infty}^{+\infty} \bar{\varphi}^{(n)}(x) e^{-\mathrm{i} \frac{n y}{R}}
$$

while the decomposition of the real field $\Phi$ is

$$
\Phi(x, y)=\sum_{n=1}^{\infty} \Phi_{-}^{(n)}(x) \sin \frac{n y}{R}+\sum_{n=0}^{\infty} \Phi_{+}^{(n)}(x) \cos \frac{n y}{R} .
$$

A similar decomposition as in (14) and (15) holds for the charged fermions $\lambda_{\phi}, \lambda_{\bar{\phi}}$ and the field $A_{5}$, respectively. In case (i) we consider spontaneous SUSY breaking. To assume in $(15) \Phi_{-}^{(n)}(x) \equiv 0$ and $\Phi_{+}^{(n)}(x)=\delta_{n 0} f(x)$ with some slowly varying function $f$ would simply shift the SUSY mass $M$ by a finite amount. We set $\Phi_{+}^{(n)}(x) \equiv 0$ when considering case (i). We are interested in the generation of a $4 \mathrm{D}$ effective one-loop potential for $\Theta$. Since the gauginos $\lambda_{1,2}$ do not couple to $A_{5}$ they are irrelevant for the Hosotani mechanism. As in (12) we assume $A_{5}$ to be constant in $y$ - only its lowest KK mode contributes. The shift of the masses $m_{n}^{\phi, \bar{\phi}}$ of the charged, bosonic KK modes can be calculated after a diagonalization of the $(2 \times 2)$-mass matrix for $\bar{\phi}_{n}$ and $\phi_{n}$ at the $n$th KK-level [15]:

$$
m_{n}^{\varphi}=m_{n}^{\bar{\varphi}}=\left[\left(\frac{n}{R}\right)^{2}+M^{2}\right]^{1 / 2} \rightarrow m_{n}^{\varphi, \bar{\varphi}, \pm}=\left[\frac{1}{R^{2}}\left(n \pm \frac{F_{T}}{2}\right)^{2}+M^{2}\right]^{1 / 2},
$$

The KK masses of charged fermions,

$$
m_{n}^{\lambda}=m_{n}^{\bar{\lambda}}=\left[\left(\frac{n}{R}\right)^{2}+M^{2}\right]^{1 / 2}
$$

are not shifted. This breaking of SUSY occurs through the non zero $F_{T}$ of the radion supermultiplet (13). Therefore, the SUSY transformation for the $\Psi_{R}^{5}$ state (the fifth component of right handed gravitino) is $\delta_{\xi} \Psi_{R}^{5} \sim \xi F_{T}$ ( $\xi$ is a Grassmann variable of the SUSY transformation). Thus, $\Psi_{R}^{5}$ emerges as a goldstino, becomes the longitudinal mode of $4 \mathrm{D}$ gravitino and gives mass to it, see [25].

Let us now consider case (ii). Here SUSY breaking proceeds by a non vanishing scalar VEV of $\partial_{5} \Phi$ and therefore, the latter appears as an order parameter for SUSY breaking. One can see from (10) that, $\langle D\rangle=-\left\langle\partial_{5} \Phi\right\rangle$ (since $\langle\varphi\rangle=\langle\bar{\varphi}\rangle=0$ ) and $\left\langle\partial_{5} \Phi\right\rangle \neq 0$ indicates complete SUSY breaking through the $D$-term. Recalling the $4 \mathrm{D} \mathcal{N}=1$ SUSY transformation for the gaugino

$$
\delta_{\xi} \lambda_{1}=\mathrm{i} D \xi+\sigma^{\mu \nu} A_{\mu \nu} \xi
$$


we see that in this case the $\lambda_{1}$ gaugino appears as a goldstino. For simplicity we assume in (15)

$$
\Phi_{-}^{(n)}(x) \equiv \delta_{n 1} V, \quad \Phi_{+}^{(n)}(x) \equiv 0 .
$$

This induces next and next-to-next neighbor interactions in the fluctuating KK tower, see Appendix A. Correspondingly, higher KK modes of $\Phi$ would cause non vanishing elements of the KK mass matrix further away from the diagonal. The field expectation value $V$ is not dynamical. ${ }^{2}$. For the one-loop potential $\mathcal{V}^{\text {eff }}(\Theta)$ to be relevant for inflation, we assume here that some external dynamics keeps $V(x)$ constant and away from zero and that the overall vacuum energy vanishes at treelevel. This would happen, for example, if a SUSY-breaking potential $P(V)$ was added to the effective $4 \mathrm{D}$ dynamics such that the sum of the tree-level potential $\sim R^{-2} V^{2}$ and $P(V)$ has a nontrivial minimum at zero energy. Since only very particular potential couplings are allowed by 5D SUSY, one can induce $P(V)$ on the $4 \mathrm{D}$ level. This would require an orbifold scenario with appropriate brane potential couplings. The calculation of the effective potential for a Wilson-line phase within an orbifold construction is beyond the scope of this paper [22].

\section{$3 \quad \mathcal{V}^{\text {eff }}(\Theta)$ from $F$-term SUSY breaking}

At tree-level the Wilson-line phase $\Theta$ would not have a potential in 4D due to 5D gauge invariance. For a compactified extra dimension this is no longer true if the quantum fluctuations of charged bulk matter are integrated out [9]. We assume that w.r.t. $4 \mathrm{D}-x\left\langle A_{5}\right\rangle$ varies on scales larger than the compactification scale $R^{-1}$ such that the lowest order in a derivative expansion of the quantum effective potential in $4 \mathrm{D}$ is a good approximation.

When calculating the one-loop effective potential in 4D for $\Theta$ the presence of the $A_{5}$ coupling to $\varphi^{(n)}, \bar{\varphi}^{(n)}$ and $\lambda_{\phi}, \lambda_{\bar{\phi}}$ in $(10)$ and the fact that $F_{T} \neq 0$ effectively modify the KK masses as follows

$$
\begin{aligned}
m_{n}^{\varphi, \bar{\varphi}, \pm} & =\left[\frac{1}{R^{2}}\left(n+\frac{q \Theta}{2 \pi} \pm \frac{1}{2} F_{T}\right)^{2}+M^{2}\right]^{1 / 2} \\
m_{n}^{\lambda}=m_{n}^{\bar{\lambda}} & =\left[\frac{1}{R^{2}}\left(n+\frac{q \Theta}{2 \pi}\right)^{2}+M^{2}\right]^{1 / 2} .
\end{aligned}
$$

After a Schwinger parametrization of tr log we have

$$
\mathcal{V}^{\mathrm{eff}}(\Theta)=-\frac{1}{(4 \pi)^{2}} \mathcal{S} \operatorname{Tr} \int_{0}^{\infty} \frac{d t}{t^{3}} \exp (-\mathcal{M} t)
$$

\footnotetext{
${ }^{2}$ On the one hand, slow-roll for $V$ due to $4 \mathrm{D}$ tree-level dynamics $\left(V^{2}\right.$ potential) would require $V \gg M_{P}$ which is precisely what we would like to avoid. On the other hand, loop effects, which qualitatively change the tree-level potential for $V$ such that $V$ is slowly rolling at $V<M_{P}$, are not under control in a perturbative treatment of the supertrace appearing in the computation of the Hosotani potential for $\Theta$.
} 


$$
\begin{gathered}
=-\frac{1}{(4 \pi)^{2}} \sum_{n=-\infty}^{\infty} \int_{0}^{\infty} \frac{d t}{t^{3}} \exp \left(-M^{2} t\right)\left\{\exp \left[-\frac{t}{R^{2}}\left(n+\frac{q \Theta}{2 \pi}+\frac{F_{T}}{2}\right)^{2}\right]+\right. \\
\left.\exp \left[-\frac{t}{R^{2}}\left(n+\frac{q \Theta}{2 \pi}-\frac{F_{T}}{2}\right)^{2}\right]-2 \exp \left[-\frac{t}{R^{2}}\left(n+\frac{q \Theta}{2 \pi}\right)^{2}\right]\right\},
\end{gathered}
$$

where $\mathcal{S} \operatorname{Tr}$ and $\mathcal{M}$ denote the supertrace and the (diagonal) super mass matrix for the fluctuating bosonic and fermionic fields, respectively. As usual ${ }^{3}$, we perform a Poisson resummation,

$$
\sum_{n=-\infty}^{+\infty} e^{-\alpha^{2}(n+\sigma)^{2}}=\frac{\sqrt{\pi}}{\alpha} \sum_{k=-\infty}^{+\infty} e^{-\frac{\pi^{2} k^{2}}{\alpha^{2}}} e^{2 \mathrm{i} \pi k \sigma},
$$

and afterwards carry out the $t$ integral in (21). The result of the integration is $2 M^{5} K_{5 / 2}(2 \pi R M k)$ where $K_{5 / 2}$ denotes a modified Bessel function. Collecting everything, we finally obtain

$$
\begin{aligned}
\mathcal{V}^{\mathrm{eff}}(\Theta)= & \frac{3}{16 \pi^{6}} \frac{1}{R^{4}} \sum_{k=1}^{\infty} \exp (-2 \pi k R M)\left[1-\cos \left(\pi k F_{T}\right)\right] \times \\
& \frac{\cos (q k \Theta)}{k^{5}}\left[1+2 \pi k R M+\frac{1}{3}(2 \pi k R M)^{2}\right],
\end{aligned}
$$

in complete agreement with a world-line calculation (Appendix B). Notice that the divergent bosonic and fermionic contributions at $k=0$ cancel exactly because of SUSY - the usual fine-tuning of the cosmological constant is not needed. For $F_{T} \neq 0$ and small $\Theta$ the effective potential is positive, and thus it drives de Sitter expansion if $\Theta$ is rolling sufficiently slowly. In the limit of unbroken SUSY, $F_{T} \rightarrow 0$, the effective one-loop potential for $\Theta$ vanishes.

\section{$4 \mathcal{V}^{\text {eff }}(\Theta)$ from $D$-term SUSY breaking}

Let us now investigate case (ii), namely the effect of $D$-term SUSY breaking according to (19). The one-loop effective potential in this case reads

$$
\mathcal{V}^{\mathrm{eff}}=-\frac{1}{16 \pi^{2}} \mathcal{S} \operatorname{Tr} \int_{0}^{\infty} \frac{d t}{t^{3}}\left[e^{-(D+B) t}\right]
$$

where $\mathcal{S} \operatorname{Tr}$ also indicates the trace in KK space. The matrices $D$ and $B$ denote the diagonal and the off-diagonal parts of the KK mass-squared matrices. We will compute $\mathcal{V}^{\text {eff }}$ in quadratic order in $B$. The treatment of the term $\frac{(-1)^{l}}{l !} t^{l} \mathcal{S} \operatorname{Tr}(D+B)^{l}$ [expansion of the exponential in (24)] is presented in Appendix A. Under consideration of (A.11), the quadratic order in $B$ reads

$$
\mathcal{S} \operatorname{Tr}\left(e^{-(D+B) t}\right)=-2\left(\frac{q V}{2 R}\right)^{2} \sum_{n=-\infty}^{+\infty} \sum_{l=2}^{+\infty} \frac{(-1)^{l}}{(l-1) !} t^{l} \frac{D_{n}^{l-1}-D_{n-1}^{l-1}}{D_{n}-D_{n-1}}=
$$

\footnotetext{
${ }^{3}$ See, however, Appendix $\mathrm{B}$ where we sketch a calculation of $\mathcal{V}^{\text {eff }}(\Theta)$ using the world-line formalism.
} 


$$
2\left(\frac{q V}{2 R}\right)^{2} t \sum_{n=-\infty}^{+\infty} \frac{e^{-D_{n} t}-e^{-D_{n-1} t}}{D_{n}-D_{n-1}}=-2\left(\frac{q V}{2 R}\right)^{2} t^{2} \sum_{n=-\infty}^{+\infty} \int_{0}^{1} d y e^{-D_{n}(1-y) t-D_{n-1} y t} .
$$

We now perform a Poisson resummation (22) in (25). Recalling that

$$
D_{n}=\frac{1}{R^{2}}\left(n-\frac{q \Theta}{2 \pi}\right)^{2}+M^{2}+\frac{q^{2} V^{2}}{2},
$$

we obtain

$$
\mathcal{V}^{\mathrm{eff}}=\frac{1}{8 \pi^{5 / 2}}\left(\frac{q V}{2 R}\right)^{2} R \int_{o}^{1} d y \sum_{k=-\infty}^{+\infty} e^{-\mathrm{i} k q \Theta-2 \mathrm{i} \pi k y} I_{k}
$$

with

$$
I_{k}=\int_{0}^{\infty} \frac{d t}{t^{3 / 2}} \exp \left[-\left(M^{2}+\frac{q^{2} V^{2}}{2}+R^{-2} y(1-y)\right) t-\frac{\pi^{2} R^{2}}{t} k^{2}\right] .
$$

Performing the $t$-integration for $|k| \geq 1$, we get

$$
I_{k}=\frac{1}{\sqrt{\pi} R|k|} \exp \left[-2 \pi R|k|\left(M^{2}+q^{2} V^{2} / 2+R^{-2} y(1-y)\right)^{1 / 2}\right] .
$$

The case $k=0$ leads to a $\Theta$-independent, linear UV divergence which renormalizes the $4 \mathrm{D}$ gauge coupling (a discussion of this is presented at the end of the paper). We omit this $\Theta$ independent part when studying inflation. Thus the final expression for $\mathcal{V}^{\text {eff }}$ reads

$$
\mathcal{V}^{\mathrm{eff}}=\frac{1}{4 \pi^{2}}\left(\frac{q V}{2 R}\right)^{2} \sum_{k=1}^{+\infty} \frac{\cos (q k \Theta)}{k} \mathcal{C}_{k}
$$

where

$$
\mathcal{C}_{k}=\int_{0}^{1} d y \cos (2 \pi k y) \exp \left[-2 \pi R k\left(M^{2}+q^{2} V^{2} / 2+R^{-2} y(1-y)\right)^{1 / 2}\right] .
$$

The same result is obtained by a (very fast!) world-line method calculation in Appendix B. Notice the exponential suppression of higher winding modes in the coefficient $\mathcal{C}_{k}$. If there is a hierarchy $M R \gg 1$ the effective potential is strongly suppressed.

The effective potentials in (23), (30) both are invariant under transformations $\Theta \rightarrow \Theta+\frac{2 \pi}{q} m$ ( $m$ integer). This is an expected result. Due to the form of KK mass spectrum in (20), (A.3), (A.4), the Lagrangian is not changing under this shift because there is summation over an infinite number of KK states. This manifests the $5 \mathrm{D}$ gauge invariance.

\section{Cosmological applications}

\subsection{Slow roll and e-foldings during inflation}

To decide under what conditions $\Theta$ can drive inflation we have to look at its $4 \mathrm{D}$

kinetic term as it follows from the $5 \mathrm{D}$ gauge curvature by integrating over $y$ [6]. This 
may seem strange at first sight since we used a derivative expansion in zeroth-order approximation when calculating $\mathcal{V}^{\text {eff }}(\Theta)$. However, in the slow-roll regime derivative terms arising from the expansion of $\mathcal{V}^{\text {eff }}\left(\Theta, \partial_{\mu} \Theta\right)$ are strongly suppressed as compared to the ones coming from the 5D gauge curvature, see below. We have

$$
\mathcal{L}_{\Theta}^{4 D}=\frac{1}{2} \frac{\partial^{\mu} \Theta \partial_{\mu} \Theta}{(2 \pi R)^{2} g_{4}^{2}}-\mathcal{V}^{\mathrm{eff}}(\Theta)
$$

So the canonically normalized field $\phi_{\Theta}$ is defined as

$$
\phi_{\Theta} \equiv f_{\Theta} \Theta \equiv \frac{\Theta}{2 \pi R g_{4}}
$$

where $\left(g^{(4)}\right)^{2} \equiv \frac{\left(g^{(5)}\right)^{2}}{2 \pi R}$ and $M_{P}$ is the 4 D reduced Planck mass. The slow-roll conditions for the field $\phi_{\Theta}$ read

$$
\epsilon=\frac{\left(M_{P}\right)^{2}}{2}\left(\frac{\left(\mathcal{V}^{\mathrm{eff}}\right)^{\prime}}{\mathcal{V}^{\mathrm{eff}}}\right)^{2} \ll 1, \quad|\eta|=\left(M_{P}\right)^{2}\left|\frac{\left(\mathcal{V}^{\mathrm{eff}}\right)^{\prime \prime}}{\mathcal{V}^{\mathrm{eff}}}\right| \ll 1,
$$

where $\left(\mathcal{V}^{\text {eff }}\right)^{\prime},\left(\mathcal{V}^{\text {eff }}\right)^{\prime \prime}$ denote the potential's derivatives in respect of $\phi_{\Theta}$. Let us first discuss case (i). We only consider the contribution at $k=1$ in (23) since terms with $k>1$ are exponentially and strongly power suppressed. Substituting the $k=1$ part of (23) or (30) into (34), we obtain

$$
\begin{aligned}
\epsilon & =\frac{1}{2}\left(2 \pi q g_{4}\right)^{2}\left(M_{P} R\right)^{2} \tan ^{2}\left[2 \pi q g_{4} R \phi_{\Theta}\right] \\
|\eta| & =\left(2 \pi q g_{4}\right)^{2}\left(M_{P} R\right)^{2} .
\end{aligned}
$$

According to (35) a hierarchy between $R^{-1}$ and $M_{P}$ can be compensated by a small $4 \mathrm{D}$ gauge coupling $g_{4} \ll 1$. Moreover, a small $g_{4}$ keeps the argument of the tangent small in $\epsilon$ and thus yields an additional suppression. Let us now look at the amount of e-foldings we may expect to be produced by the slowly rolling field $\phi_{\Theta}$.

The number of e-foldings for canonically normalized field $\phi_{\Theta}($ of $\Theta$ ) is given by the following formula

$$
N_{\Theta}=\int_{t^{i}}^{t^{f}} H d t \simeq \frac{8 \pi}{M_{P}^{2}}\left|\int_{\phi_{\Theta}^{i}}^{\phi_{\Theta}^{f}} \frac{\mathcal{V}^{\mathrm{eff}}}{\left(\mathcal{V}^{\mathrm{eff}}\right)^{\prime}} d \phi_{\Theta}\right| .
$$

Using (23), (30), (33), for both scenarios considered above, we obtain

$$
N_{\Theta}=\frac{\rho}{\left(M_{P} R\right)^{2} \pi g_{4}^{2}}, \quad \rho=\frac{2}{q^{2}} \ln \left|\sin \left(2 \pi q g_{4} R \phi_{\Theta}\right)\right|_{\phi_{\Theta}^{i}}^{\phi_{\Theta}^{f}} \sim \mathcal{O}(1) .
$$

Taking in $(37) M_{P} R=10-100, g_{4} \lesssim 10^{-2}-10^{-3}$, we easily get $N_{\Theta} \gtrsim 55$, which evades the horizon problem and guarantee flatness of the Universe up to the needed accuracy. 


\subsection{Application for quintessence}

As was pointed out in [10], for a hierarchy $1 / R \ll M$ (recall that $M$ is a SUSY bulk mass for matter), the generated effective potentials are getting strongly suppressed. From (23), (30) one sees that the potentials in both scenarios (i) and (ii) aquire a factor $\sim \exp (-2 \pi M R)$, which for $M R=30-50$ provides suppression such that $\left\langle\mathcal{V}^{\text {eff }}\right\rangle \sim\left(3 \cdot 10^{-3} \mathrm{eV}\right)^{4}$. This amount can be associated with dark energy, making the model a candidate for quintessence [11].

The presented effective potentials may be relevant for both inflation and quintessence. Inflation would start at $R M \sim 1$, and at the end of inflation or after reheating the radion field would relax to the minimum of a stabilizing potential corresponding to $M R \gg 1$. This, of course, requires a dynamical model for the radion field, for its foundation within 5D supergravity see $[25,26]$.

\section{Discussion and outlook}

In this paper we have realized the idea of gauge inflation within a 5D supersymmetric setting exploiting $U(1)$ gauge symmetry. For this, two possibilities of SUSY breaking were considered. The 'no scale' one-loop potential has the advantage that the treelevel contribution vanishes. In both scenarios, the obtained effective potentials make the 'Wilson-line phase $\Theta$ ' a candidate for the inflaton. Namely, for a given hierarchy between $M_{P}$ and the compactification scale $1 / R$ a proper choice of the $4 \mathrm{D}$ gauge coupling $g_{4}$ guarantees slow roll conditions and the required number of e-foldings. Due to SUSY the fine-tuning of the $4 \mathrm{D}$ cosmological constant to zero after inflation is with respect to $\sim R^{-4}$ as compared to the the non-SUSY value of $\sim R\left(M_{P}^{(5)}\right)^{5}$ where $M_{P}^{(5)} \sim M_{P}$ denotes the $5 \mathrm{D}$ Planck scale. Also, the specific forms of the effective potentials open up the possibility to describe quintessence.

As a byproduct we have demonstrated that the winding mode representation can be written down directly using the world-line method (Appendix B).

For quantitative estimates [in eqs. (34)-(37)], one should use a perturbatively small, renormalized value of $g_{4}$. As it is known the KK excitations above $1 / R$ induce a power law running of $g_{4}$ (linear in case of 5D). This effect was observed in our calculation of $\mathcal{V}^{\text {eff }}(\Theta)$ in the case of $D$-term SUSY breaking. The emerging linear UV divergence, coming from the zero winding mode, should be absorbed into the bare $1 / g_{4}^{2}$ [the corresponding operator is $\frac{1}{g_{4}^{2}}\left(\partial_{5} \Phi\right)^{2}$ ]. This is nothing but a renormalization of the gauge coupling. Another question, which should be understood (for both scenarios), is the origin of the small value for $g_{4}$ and the mechanism which guarantees its smallness. For example, an embedding of the $U(1)$ in a non Abelian gauge symmetry, which is unbroken at high energies, prevents $g_{4}$ to reach the Landau pole. For such a scenario, one could have in mind some specific GUT, which naturally unifies $U(1)$ with the SM interactions. Another way for an ultraviolet completion is the possibility of embedding the 5D SUSY $U(1)$ gauge theory in a superstring model. 


\section{Acknowledgements}

Research of F.P.C. is supported by Fundação para a Ciência e a Tecnologia (grant $\mathrm{SFRH} / \mathrm{BD} / 4973 / 2001)$.

\section{Appendix A: KK spectrum and $\mathcal{S} \operatorname{Tr}\left[\mathrm{e}^{-(D+B) t}\right]$}

Here we quote the mass matrices for the fluctuating KK modes as they arise from the $D$-term SUSY breaking according to (19). After KK decomposition of the fields appearing in (11) and subsequent integration over $y$ the bilinear terms in the effective $4 \mathrm{D}$ bosonic KK modes are

$$
\mathcal{V}^{(2)}=\sum_{n, m=-\infty}^{+\infty}\left\{\varphi^{(n)} M_{m, n}^{2} \varphi^{(m) *}+\bar{\varphi}^{(n)} \bar{M}_{m, n}^{2} \bar{\varphi}^{(m) *}\right\} .
$$

The bilinear expressions for the superpartners have the form

$$
-\mathcal{L}_{\lambda}^{(2)}=\sum_{n, m=-\infty}^{+\infty}\left\{\lambda_{\bar{\phi}}^{(n)} M_{n, m}^{\lambda} \lambda_{\phi}^{(m)}+\bar{\lambda}_{\bar{\phi}}^{(n)} M_{n, m}^{\lambda *} \bar{\lambda}_{\phi}^{(m)}\right\} .
$$

The matrix elements appearing in (A.1) and (A.2) read as follows

$$
\begin{aligned}
M_{n, n}^{2} & =\bar{M}_{n, n}^{2}=\left(\frac{n}{R}-\frac{q \Theta}{2 \pi R}\right)^{2}+M^{2}+\frac{q^{2}}{2} V^{2}, \\
M_{n, n+1}^{2} & =-M_{n, n-1}^{2}=-\bar{M}_{n, n+1}^{2}=\bar{M}_{n, n-1}^{2}=\mathrm{i} q M V, \\
M_{n, n+2}^{2} & =M_{n, n-2}^{2}=\bar{M}_{n, n+2}^{2}=\bar{M}_{n, n-2}^{2}=-\frac{q^{2}}{4} V^{2},
\end{aligned}
$$

all other elements are zero. The matrix elements of fermionic states are

$$
M_{n, n}^{\lambda}=\mathrm{i}\left(\frac{n}{R}-\frac{q \Theta}{2 \pi R}\right)+M, \quad M_{n, n-1}^{\lambda}=-M_{n, n+1}^{\lambda}=\frac{\mathrm{i} q}{2} V,
$$

while all other elements are zero. For the calculation of the effective potential the matrix $M^{\lambda} M^{\lambda+} \equiv\left(M^{2}\right)^{\lambda}$ is relevant where $M^{\lambda+}$ denotes complex conjugation of $M^{\lambda}$ and transposition in the KK indices. The elements of $\left(M^{2}\right)^{\lambda}$ are

$$
\begin{aligned}
\left(M^{2}\right)_{n, n}^{\lambda} & =\left(\frac{n}{R}-\frac{q \Theta}{2 \pi R}\right)^{2}+M^{2}+\frac{q^{2}}{2} V^{2}, \quad\left(M^{2}\right)_{n, n+2}^{\lambda}=\left(M^{2}\right)_{n+2, n}^{\lambda}=-\frac{q^{2}}{4} V^{2}, \\
\left(M^{2}\right)_{n, n+1}^{\lambda} & =-\mathrm{i} q M V-\frac{q V}{2 R}, \quad\left(M^{2}\right)_{n+1, n}^{\lambda}=\mathrm{i} q M V-\frac{q V}{2 R} .
\end{aligned}
$$

All other elements are zero.

Let us now evaluate the expression

$$
\mathcal{S} \operatorname{Tr} e^{-(D+B) t}=\mathcal{S} \operatorname{Tr} \sum_{l=0}^{+\infty} \frac{(-1)^{l}}{l !} t^{l}(D+B)^{l} .
$$


We have

$$
(D+B)^{l}=D^{l}+\sum_{p=0}^{l-1} D^{l-1-p} B D^{p}+\sum_{p=0}^{l-2} \sum_{q=0}^{l-2-p} D^{l-2-p-q} B D^{p} B D^{q}+\cdots .
$$

The supertrace of the first term in (A.7) vanishes because the elements of $D$ coincide for scalars and fermions, see (A.3) and (A.5)]. The trace of the second term in (A.7) is zero because $B$ is an off-diagonal matrix. Therefore the leading contributions are due to the third term in (A.7). We have

$$
\begin{gathered}
\operatorname{Tr}\left(D^{l-2-p-q} B D^{p} B D^{q}\right)=\operatorname{Tr}\left(D^{l-2-p} B D^{p} B\right)= \\
\sum_{n=-\infty}^{+\infty}\left(D_{n}^{l-2-p} D_{n \pm 1}^{p}\left|B_{n, n \pm 1}\right|^{2}+D_{n}^{l-2-p} D_{n \pm 2}^{p}\left|B_{n, n \pm 2}\right|^{2}\right) \\
\mathcal{S} \operatorname{Tr}\left(D^{l-2-p-q} B D^{p} B D^{q}\right)=-2\left(\frac{q V}{2 R}\right)^{2} \sum_{n=-\infty}^{+\infty} D_{n}^{l-2-p}\left(D_{n-1}^{p}+D_{n+1}^{p}\right) .
\end{gathered}
$$

According to (A.7), (A.9) we evaluate the following sum

$$
\begin{gathered}
\sum_{n=-\infty}^{+\infty} \sum_{p=0}^{l-2} \sum_{q=0}^{l-2-p} D_{n}^{l-2-p}\left(D_{n-1}^{p}+D_{n+1}^{p}\right)=\sum_{n=-\infty}^{+\infty} \sum_{p=0}^{l-2}(l-1-p) D_{n}^{l-2-p}\left(D_{n-1}^{p}+D_{n+1}^{p}\right)= \\
\sum_{n=-\infty}^{+\infty} \sum_{p=0}^{l-2}(l-1-p) D_{n}^{l-2-p} D_{n-1}^{p}+\sum_{n=-\infty}^{+\infty} \sum_{p=0}^{l-2}(1+p) D_{n}^{l-2-p} D_{n-1}^{p}= \\
\sum_{n=-\infty}^{+\infty} \sum_{p=0}^{l-2} l D_{n}^{l-2-p} D_{n-1}^{p}=\sum_{n=-\infty}^{+\infty} l \frac{D_{n}^{l-1}-D_{n-1}^{l-1}}{D_{n}-D_{n-1}} .
\end{gathered}
$$

We have obtained the second term in the second line of (A.10) by substituting $p \rightarrow l-2-p$ and shifting $n \rightarrow n-1$. Finally, we have

$\mathcal{S} \operatorname{Tr}(D+B)^{l}=-2\left(\frac{q V}{2 R}\right)^{2} \sum_{n=-\infty}^{+\infty} \sum_{p=0}^{l-2} l D_{n}^{l-2-p} D_{n-1}^{p}=-2\left(\frac{q V}{2 R}\right)^{2} \sum_{n=-\infty}^{+\infty} l \frac{D_{n}^{l-1}-D_{n-1}^{l-1}}{D_{n}-D_{n-1}}$.

\section{Appendix B: Calculation of $\mathcal{V}^{\mathrm{erf}}(\Theta)$ in world-line for- malism}

Here we briefly sketch how an effective one-loop potential for $\Theta$ arises in the worldline formalism. First, we consider bosonic fluctuations. Like in thermal physics [23] the effective one-loop potential in the world-line formalism is expressed in terms of 
an integration over the proper length of all possible closed-path trajectories. We have

$$
\begin{aligned}
\int d^{4} x \mathcal{V}^{\mathrm{eff}}(\Theta)= & -\int d^{5} x \int_{0}^{\infty} \frac{d T}{T} \sum_{k=-\infty}^{\infty} \int \mathcal{D}^{5} y \\
& \exp \left[-\int_{0}^{T} d \tau\left(\frac{\left(\dot{x}_{M}\right)^{2}}{4}+i q \dot{x}_{5} A_{5}+M^{2}\right)\right],
\end{aligned}
$$

where

$$
x_{M}(\tau)=2 \pi R k \frac{\tau}{T} \delta_{M 5}+y_{M}(\tau)+x_{M}, \quad \int_{0}^{T} d \tau y_{M}(\tau)=0 .
$$

In (B.2) a constant part $x_{5}$ and a non constant, periodic part $y(\tau)$ have been separated from the topological part of the trajectory. Performing the integration over all trajectories and using $\Theta=2 \pi R A_{5}$, we arrive at

$$
\begin{gathered}
\int d^{4} x \mathcal{V}^{\mathrm{eff}}(\Theta)=-\int d^{5} x \sum_{k=-\infty}^{\infty} \exp [i k q \Theta] \int_{0}^{\infty} \frac{d T}{T} \frac{1}{(4 \pi T)^{5 / 2}} \exp \left[-\frac{(\pi R k)^{2}}{T}-M^{2} T\right] \\
=-\int d^{4} x \frac{3}{16 \pi^{6}} \frac{1}{R^{4}} \sum_{k=1}^{\infty} \exp (-2 \pi n R M) \frac{\cos (k q \Theta)}{k^{5}}\left[1+2 \pi k R M+\frac{1}{3}(2 \pi k R M)^{2}\right] \\
-\int d^{5} x \int_{1 / \Lambda^{2}}^{\infty} \frac{d T}{T} \frac{1}{(4 \pi T)^{5 / 2}} \exp \left(-M^{2} T\right) .
\end{gathered}
$$

The last term in (B.3) is a contribution from $k=0$ and is quintically UV divergent. It expresses the usual cosmological-constant problem in 5D. Whether a cutoff must be introduced or not in the KK tower needs not be addressed in the world-line formulation. In particular in the non-SUSY case we do, however, need a 5D cutoff $\Lambda^{-2} \leq T$ for the zero-winding contribution.

In case (i) (spontaneous, no-scale SUSY breaking) the coupling to the gauge field of the two complex, fluctuating scalars and the two Dirac fermions is in the world-line formalism described by

$$
\mathrm{i} \dot{x}_{5}\left(\begin{array}{cc}
q A_{5} & \frac{\mathrm{i}}{2 R} F_{T} \\
-\frac{\mathrm{i}}{2 R} F_{T} & q A_{5}
\end{array}\right), \quad \mathrm{i} q \dot{x}_{5} A_{5},
$$

respectively. Using these interactions to calculate the effective potential in analogy to (B.1) and taking a trace, we again arrive at expression (23) which was obtained from a Poisson resummation of the KK spectrum.

In case (ii) ( $D$-term SUSY breaking by $\left.\Phi=V \sin \frac{y}{R}\right)$ the world-line treatment is more involved since now the 'outer' field $\Phi$ is not constant [24]. Let us briefly sketch how things work here. For charged scalars we only have to shift $M \rightarrow M-\frac{q}{2} \Phi$ in 
(B.1). For Dirac fermions interacting with $\Phi$ the path integral in (B.1) is enlarged by a world-line Grassmann integration

$$
\int \mathcal{D} \psi_{6} \exp \left(-\int_{0}^{T} d \tau\left(\frac{1}{2} \psi^{M} \dot{\psi}_{M}+\frac{1}{2} \psi_{6} \dot{\psi}_{6}-2 i \psi_{6} \psi_{5} \partial_{5} \Phi\right)\right.
$$

The additional component $\psi_{6}$ is needed if the coupling of the spinning particle to the scalar background $\Phi$ is of the Yukawa-type in field theory [24]. Since the part in (B.1), which depends on $x_{5}$ is universal for fermionic and bosonic fluctuations we only need to consider the term $q i \psi_{6} \psi_{5} \partial_{5} \Phi$ in (B.5). This term breaks the supersymmetric cancellation. We write $\Phi=V /(2 i)\left(\exp \left[i x_{5}(\tau) / R\right]-\exp \left[-i x_{5}(\tau) / R\right]\right)$, substitute this into (B.5) and expand the exponential in (B.5) up to second order in $V$. Performing the Grassmann-integration and exploiting translational invariance on the circle, the part of (B.5) due to $q i \psi_{6} \psi_{5} \partial_{5} \Phi$ turns into

$$
-2 T \frac{V^{2}}{R^{2}} \int_{0}^{T} d \tilde{\tau} G_{F}^{2}(\tilde{\tau}) \exp \left(-G_{B}(\tilde{\tau}) / R^{2}\right) \cos \left(2 \pi k \frac{\tilde{\tau}}{T}\right)
$$

where $G_{F}=\operatorname{sign} \tilde{\tau}$ and $G_{B}=\tilde{\tau}(1-\tilde{\tau}) / T$ denote the fermionic and bosonic worldline Green's functions, respectively. Substituting $\tilde{\tau}=y T$ in (B.6), performing the $T$ - and the $x^{5}$-integrations, and neglecting the $\Phi$ dependent contributions from the

bosonic sector and the linearly UV divergent $k=0$ contribution, we arrive at the following effective potential

$$
\frac{(q V)^{2}}{16 \pi^{2} R^{2}} \sum_{k=1}^{\infty} \frac{\cos (k q \Theta)}{k} \int_{0}^{1} d y \cos (2 \pi k y) \exp \left(-2 \pi k\left(M^{2} R^{2}+y(1-y)\right)^{1 / 2}\right) .
$$

This is the formula one would obtain from (30) by isolating the quadratic order in $V$.

\section{References}

[1] A.H. Guth, Phys. Rev.D23, 347 (1981);

A.D. Linde, Phys. Lett.B 108, 389 (1982).

[2] E. Copeland et al., Phys. Rev. D 49, 6410 (1994);

G. Dvali, Q. Shafi, R. Schaefer, Phys. Rev. Lett. 73 , 1886 (1994).

[3] N. Arkani-Hamed, A. G. Cohen, and H. Georgi, Phys. Rev. Lett. 86, 4757 (2001) [hep-th/0104005];

C. T. Hill, S. Pokorski, J. Wang, Phys. Rev. D64, 105005 (2001) [hepth/0104035];

N. Arkani-Hamed, A.G. Cohen, E. Katz, A.E. Nelson, T. Gregoire, and J.G. Wacker, JHEP 0208, 021 (2002);

I. Low, W. Skiba, and D. Smith, Phys. Rev. D66, 072001 (2002) [hepph/0207243]; D. E. Kaplan and M. Schmaltz, hep-ph/0302049. 
[4] S. Mollerach, Phys. Rev. D42, 313 (1990);

D.H. Lyth and D. Wands, Phys. Lett. B524, 5 (2002) [hep-ph/0110002];

T. Moroi and T. Takahashi, Phys. Lett. B522, 215 (2001) [hep-ph/0110096];

K. Dimopoulos, D.H. Lyth, A. Notari, and A. Riotto, hep-ph/0304050.

[5] K. Freese, J.A. Friemann, and A.V. Olinto, Phys. Rev. Lett. 65, 3233 (1990);

F.C. Adams, J.R. Bond, K. Freese, J.A. Friemann, and A.V. Olinto, Phys. Rev.D47, 426 (1993) [hep-ph/9207245].

[6] N. Arkani-Hamed, H.-C. Cheng, P. Creminelli, and L. Randall, hep-th/0302034, hep-th/0301218;

D. Kaplan and N. Weiner, hep-ph/0302014.

[7] B. Feng, M. Li, R.-J. Zhang, and X. Zhang, astro-ph/0302479.

[8] C.L. Bennett et al., astro-ph/0302207.

[9] Y. Hosotani, Phys. Lett.B 126, 309 (1983); hep-ph/0303066.

[10] L. Pilo, D.A.J. Rayner, and A. Riotto, hep-ph/0302087.

[11] C. Wetterich, Nucl. Phys. B302, 668 (1988);

B. Ratra, P.J. Peebles, Phys. Rev. D37, 3406 (1988);

R.R. Caldwell, R. Dave, P.J. Steinhardt, Phys. Rev. Lett. 80, 1582 (1998).

[12] I. Antoniadis, Phys. Lett. B246, 377 (1990);

R. Barbieri, L.J. Hall, Y. Nomura, Phys. Rev. D63, 105007 (2001) [hep$\mathrm{ph} / 0011311]$

N. Arkani-Hamed, L.J. Hall, Y. Nomura, D.R. Smith, and N. Weiner, Nucl. Phys. B605, 81 (2001) [hep-ph/0102090];

I. Antoniadis, K. Benakli, and M. Quiros, New J. Phys. 3, 20.1-20.24 (2001) [hep-th/0108005];

G. von Gersdorff, N. Irges, and M. Quiros, Nucl. Phys. B635, 127 (2002) [hepth/0204223], hep-th/0206029.

[13] D.M. Ghilencea and H.-P. Nilles, Phys. Lett. B507, 327 (2001) [hep$\mathrm{ph} / 0103151]$

D.M. Ghilencea, H.-P. Nilles, and S. Stieberger, New J.Phys.4, 15 (2002) [hepth/0108183];

D.M. Ghilencea, S. Groot Nibbelink, and H.-P. Nilles, Nucl. Phys. B619, 385 (2001) [hep-th/0108184].

[14] M.J. Strassler, Nucl. Phys. B385, 145 (1992);

M.G. Schmidt and C. Schubert, Phys. Lett. B318, 438 (1997); ibid B331, 69 (1994); Phys. Rev. D53, 2150 (1996);

M. Reuter, M.G. Schmidt, and C. Schubert, Ann. Phys. 259, 313 (1997). 
[15] D. Marti and A. Pomarol, Phys. Rev.D64, 105025 (2001) [hep-th/0106256].

[16] N. Arkani-Hamed, T. Gregoire, and J. Wacker, JHEP 0203, 055 (2002) [hepth/0101233];

A. Hebecker, Nucl. Phys. B632, 101 (2002) [hep-ph/0112230].

[17] E. Cremmer, S. Ferrara, C. Kounnas, D.V. Nanopoulos, Phys. Lett. B133, 61 (1983);

[18] E. Witten, Phys. Lett. B155, 151 (1985);

U. Ellwanger and M. G. Schmidt, Nucl. Phys. B294, 445 (1987);

S. Ferrara, C. Kounnas, M. Porrati, Phys. Lett. B181, 263 (1986);

S. Ferrara, C. Kounnas, and F. Zwirner, Nucl. Phys. B429, 589 (1994);

E. Dudas and C. Grojean, Nucl. Phys. B507, 553 (1997).

[19] N. Chang, S. Ouvry, and X. Wu, Phys. Rev. Lett. 51, 327 (1983).

[20] U. Ellwanger, N. Dragon, and M. G. Schmidt, Phys. Lett. B145, 192 (1984); Nucl. Phys.B 255, 544 (1984); Prog. Part. Nucl. Phys. 18, 1 (1987).

[21] M. Faibarn, L. Lopez-Honorez, and M.H.G. Tytgat, hep-ph/0302160.

[22] R. Hofmann, F. Paccetti Correia, M.G. Schmidt, Z. Tavartkiladze, In preparation.

[23] D.G.C. McKeon and A. Rebhan, Phys. Rev. D47, 5487 (1993);

M. Haack and M.G. Schmidt, Eur. Phys. J. C7, 149 (1999).

[24] M. Mondragon, L. Nellen, M.G. Schmidt, and Ch. Schubert, Phys. Lett. B351, 200 (1995) [hep-th/9502125];

ibid B366, 212 (1996) [hep-th/9510036].

[25] G.v. Gersdorff and M. Quiros, Phys. Rev. D65, 064016 (2002) [hepth/0110132];

G.v. Gersdorff, M. Quiros, and A. Riotto, Nucl. Phys. B634, 90 (2002) [hepth/0204041].

[26] For a recent formulation of 5D SUGRA see: M. Zucker, Nucl. Phys. B570, 267 (2000) [hep-th/9907082], Phys. Rev. D64, 024024 (2001) [hep-th/0009083];

T. Kugo and K. Ohashi, Prog. Theor. Phys. 108, 203 (2002) [hep-th/0203276]. 